\title{
Singular features of fertilization and their impact on the male reproductive system in eutherian mammals
}

\author{
J Michael Bedford \\ University, New York, New York 10021, USA \\ Correspondence should be addressed to J M Bedford; Email: mbedford@med.cornell.edu
}

Department of Obstetrics and Gynecology, The Center for Reproductive Medicine, Weill Medical College of Cornell

\begin{abstract}
Therian (marsupial and eutherian) mammals have evolved a suite of novel reproductive features - seen variously in their gametes, the steps of fertilization and the male reproductive tract - whose adaptive significance remains unclear. Present evidence for the betterunderstood eutherian mammals suggests that the 'prime mover' in their evolution has been the character of the egg coat, with other such features being adaptations to the consequences of this. Its elastic thickness allows the zona pellucida to stretch to a variable degree and yet remain around the blastocyst during much or all of its expansion before implantation, but its character represents an unusual challenge for spermatozoa. Novel aspects of the acrosome related to this challenge enable it to maintain a relatively prolonged binding after the onset of the acrosome reaction, and the structure, shape and behaviour of the sperm head point to physical thrust as a major element of zona penetration - with the unique configuration of gamete fusion as a sequela of this strategy. In the male, such adaptations are reflected in sperm head formation in the testis and in sperm maturation in the epididymis involving at least the sperm head's structure, plasmalemma and acrosome. This complexity allied to a slow epididymal sperm transport, a relatively modest sperm production and the brief life span of mature spermatozoa kept above the cauda epididymidis could account for the evolution of the sperm storage function - a development seemingly linked, in turn, to the need for sperm capacitation and scrotal evolution.

Reproduction (2014) 147 R43-R52
\end{abstract}

\section{Introduction}

Among the invertebrates and vertebrates used to study fertilization, it has been most difficult to unravel the mechanisms involved in this process in therian (marsupial and placental/eutherian) mammals. One problem has been the fact that their gametes and the male reproductive tract display a suite of novel features whose underlying significance remains in doubt. The thinking in early investigations of fertilization in Eutheria was based, to a considerable degree, on principles established in invertebrate models. Thus, in pioneering experiments on in vitro fertilization, Thibault \& Dauzier (1960) first washed rabbit oocytes to avoid supposed fertilizin/antifertilizin issues raised by invertebrate studies. In a similar vein, fissures ahead of sperm heads penetrating the rabbit, pig and sheep zona pellucida (see Dziuk \& Dickman (1965)) were thought to be acrosomal filaments comparable to those in Arbacia and Saccoglossus. However, it is now apparent that fertilization in Eutheria is marked by a strategy of egg penetration that diverges markedly from that in other metazoans.

Perhaps most striking in this regard is that, after negotiating the egg coat, eutherian spermatozoa do not fuse conventionally with the oolemma via the apex of the inner acrosomal membrane (IAM), but rather fuse by the mid-region of the sperm head, followed by the egg's 'phagocytosis' of the acrosomal domain (Yanagimachi \& Noda 1970a, Bedford 1972). Underlying this mode, the eutherian acrosome alone displays an attenuated stable posterior region - the equatorial segment, which is unique to eutherian mammals and whose significance lies in its ability to preserve a section of fusogenic plasma membrane overlying it (Bedford et al. 1979). An earlier puzzle was the finding that in order to fertilize, eutherian spermatozoa in the female reproductive tract must first undergo capacitation - now understood as a functional change in the sperm plasmalemma. However, there has been surprisingly little speculation as to the implication of these departures from the vertebrate norm or about other uniquely eutherian sperm head features that include a flattened form, a keratinoid nucleus and perinuclear theca, a stable IAM and an acrosomal content characterized by an insoluble matrix that persists after the acrosome reaction (AR). Equally puzzling novelties present in the male reproductive tract include a more complex sperm maturation process in the epididymis, whose terminal region - the cauda epididymidis - performs a sperm storage function regulated acutely by androgens and often the low temperature of 
the scrotum, the latter seen only in therian mammals and some passerine birds.

In considering the adaptive significance of these features, and why they have arisen, I cite a variety of evidence for the likelihood that they are related to one or another, with the prime mover being the particular character of the egg. Very small compared to that of other amniotes, and without the genetic base for yolk synthesis, the eutherian egg is invested at ovulation by the cells of the cumulus oophorus and - key to the present discussion - an unusually substantial egg coat. The discussion refers on occasion to therian mammals since marsupials exhibit a number of similar traits in this regard, and it appears that a relatively prominent if lesser egg coat has influenced sperm head design in marsupials too. However, I focus on Eutheria because the larger therian theme is complicated by a different sperm head design in marsupials, by the fact that the zona and the sperm/egg interactions differ in important respects, and because too little is known yet in marsupials about many aspects of fertilization including capacitation.

\section{The egg vestments}

The cumulus oophorus investing the zona pellucida is unique to eutherian mammals, being absent even from the comparably small eggs of marsupials, but it is hard to be sure of its significance. The post-ovulatory life of this investment of cells varies from a very few hours in some ungulates to $>24 \mathrm{~h}$ in some bats and certain insectivores in which it presents as a dense mass. Nonetheless, in keeping with its name, the cumulus exists more commonly as a cloud of cells suspended within a hyaluronic acid matrix, and we have observed in the rat that it acts to sequester into the vicinity of the eggs the very few spermatozoa that reach the often-arborized or spacious ampulla of the fallopian tube. Although eggs can be fertilized readily in the absence of the cumulus in most of the species tested, this cell mass is the essential inducer of the AR in shrews and probably some other insectivores (Bedford et al. 2004). Moreover, spermatozoa often react within the cumulus also in laboratory mammals (e.g. hamsters and rabbits), and in mice at least, such spermatozoa later penetrate the zona and fertilize (Jin et al. 2011).

From a comparative perspective, the eutherian zona pellucida is unusually formidable in a physical sense. Yet, notwithstanding its robust character, the eutherian zona has generally been viewed simply as a rather prominent egg coat, and the detection of acrosin in the rabbit acrosome in the late 1960s solidified the expectation that sperm penetrate it in part by means of acrosomal lysins. However, the eutherian zona and the mode of its penetration are anything but conventional. Approximately an order of magnitude thicker than that in most of the invertebrates and vertebrates, including some marsupials, its three or four constituent glycoproteins, first probed by
Paul Wassarman and colleagues are linked to a degree by-S-S- bonds. Moreover, not only does it display an elastic rigidity (Green 1997), but it also proves to be relatively insensitive to proteases. We have observed that the marsupial zona disappears in some 2-8 s on exposure to $1.0-0.5 \%$ trypsin at $\mathrm{pH} 7.2$, whereas that of most eutherians shows no visible change for 2 min or more.

As discussed below, the overall picture points to the physical character of the eutherian zona pellucida as the driver of special strategies for fertilization, which are reflected in the behaviour and design of the spermatozoon.

\section{Zona binding}

Throughout the animal kingdom, the binding of the sperm to the egg coat, generally brief, is often effected by the linking of single proteins in the acrosome to complementary molecules on the egg. However, the key factors involved in eutherian mammals have proven difficult to pin down. Electron microscope images obtained in eutherian mammals such as hamsters and rabbits (Barros et al. 1967, Yanagimachi \& Noda 1970b, Bedford 1972, Yanagimachi \& Phillips 1984), man and also domestic animals (e.g. Szollosi \& Hunter (1978)) demonstrate that binding is normally mediated by the fenestrated carapace of the reacted acrosome and probably involves not only a proteoglycan-related sperm surface receptor but also elements of the acrosome matrix (Jansen et al. 1995). However, it has been shown that rabbit and mouse perivitelline spermatozoa free of the reacted carapace can penetrate the zona (Kuzan et al. 1984, Inoué et al. 2011) and that there are natural variants that differ from the hamster/rabbit pattern. For example, spermatozoa of the shrews studied lose the acrosome well before zona binding (Bedford et al. 2004), and those of the guinea pig do so before they can bind to and penetrate the zona (Huang et al. 1981). In addition, although studies in the mouse describe its AR as involving the point membrane fusions that eventually translate to a vesiculated appearance (Thompson et al. 1974, Anderson et al. 1975), it is hard to find visual evidence of this or that this transient membrane complex acts as the link in zona binding. Indeed, as stated for the mouse by Zamboni (1971), 'at the time of the earliest contact between gametes, the fertilizing spermatozoon lacks the acrosome'. If spermatozoa of the mouse do indeed finally bind to the zona rather by way of the IAM, this has wider implications. It may explain the difficulty in using fertilization as the end point in this model species to identify an essential receptor on the sperm plasmalemma (see Shur (2008)) or among candidates (e.g. proacrosin, zonadhesin, P56 and arylsulfatase A) in the acrosomal matrix (see Tardif et al. (2010) and Avelia et al. (2013)).

Regardless of these last examples, it would appear that the physiological norm in most eutherian mammals 
examined thus far is represented by the hamster/rabbit mode of zona binding. However, in first describing the AR (Barros et al. 1967), it did not strike us that, in contrast to the acrosome in many metazoans, the vesiculated eutherian acrosome is unusually persistent, reflecting a stability that may depend in part on elements of the insoluble matrix. This stability allows the hyperactivated spermatozoon to maintain a juxtaposition with the zona until it begins to penetrate and as such would seem to represent a feature that is adaptive for the unusually physical challenge presented by the eutherian zona.

\section{Sperm head design, zona penetration and gamete fusion}

There is no consensus yet as to the means by which fertilizing spermatozoa cleave a path through the eutherian zona pellucida. In most metazoans, penetration of the egg coat involves either its rapid focal lysis by sperm head enzymes or, less often, the dissociative non-enzymatic action of an acrosomal component - as seen, for example, in the abalone (Lewis et al. 1982). Initially, the demonstration by Srivastava et al. (1965) that an acrosome extract could eventually destabilize the rabbit zona seemed to support the expectation that lysis is a key to sperm penetration of the eutherian egg coat as well. However, much of the evidence emerging since then undermines this view.

In addition to the zona's relative insensitivity to proteases, noted earlier, and the reports that acrosomefree spermatozoa released from the perivitelline space can fertilize, a litany of current evidence calls into question the common assumption that one or more acrosomal lysins facilitate the penetration of the eutherian zona. First, protease inhibitors that prevent fertilization in vitro also prevent the completion of the AR (Meizel 1984, llanos et al. 1993). Moreover, as seen using the transmission electron microscope, not only does the soluble content of the acrosome disperse before any inroad into the zona, but its release does not induce local erosion of the zona surface, and spermatozoa lacking an acrosome no longer display any ability to digest a gelatin substrate. Consistent with this, and despite recent claims to the contrary, the molecular structure of acrosin, the first candidate lysin, does not permit it to bind to the IAM (Klemm et al. 1991), and it has been shown that acrosin does not remain on the IAM after the AR or during the penetration of the zona pellucida (Kopecny \& Flechon 1987). Furthermore, mouse spermatozoa null for the acrosin gene can penetrate the zona (Baba et al. 1994), though more slowly as a probable result of delayed acrosomal dehiscence. Finally, sperm penetration of the rabbit zona was in no way hindered by a $\sim$ tenfold wheat germ agglutinin (WGA)-mediated increase in its resistance to trypsin (Bedford \& Cross 1978) or by the increase brought by fertilization itself (Overstreet \& Bedford 1974). Linked to this as a seeming paradox for a scenario of zona lysis, the environment of the fallopian tube effects a major increase in the resistance of the zona to pronase (Mondejar et al. 2013).

Despite such evidence, it apparently remains hard to accept that sperm penetration of the eutherian zona may not involve lysis, as reflected in several different alternatives proposed recently (e.g. Kim et al. (2008), Moreno et al. (2011), Sutovsky (2011), Xu et al. (2012) and Ferrer et al. (2012)). Nevertheless, the highly derived design of the eutherian sperm head does imply a mode of penetration in which physical factors dominate. On entering the zona matrix, the sperm head's flat profile allows the hyperactivated spermatozoon to oscillate in the scything fashion observed, and its stiffness brought about by -S-S- cross-linking of the nucleus and perforatorium ensures that the head remains erect in doing so. Not least, the IAM that bears the immediate physical brunt of this interaction has a crystalline structure (Huang \& Yanagimachi 1985) that underlies an unusual stability. However, while the IAM's physical quality precludes the fusogenic role that it serves in other groups, that role is substituted in Eutheria by the plasmalemma preserved over the inert equatorial segment of the acrosome (Bedford et al. 1979; Fig. 1). This interpretation seems to be validated by the picture in a deviant group, epitomized by the bandicoot rat, Bandicota indica, for this is characterized by a relatively thin protease-sensitive zona, by a sperm head with a circular profile and by a large bulbous acrosome that lacks an equatorial segment (Dorman et al. 2014) features consistent with a lytic mode of zona penetration. Interestingly, with its relatively broad profile and the relatively low cysteine content of its protamine and absence of perforatorium, the human sperm head is among the least well-adapted with regard to physical thrust - a design perhaps linked to the finding by Mondejar et al. (2013) that, as an exception, the human zona is not 'hardened' by the tubal environment.

In summary, the broad picture suggests that in response to a robust zona, the eutherian sperm head has evolved features that permit a necessarily prolonged binding to and then penetration of the zona by a mode in which physical factors may dominate. But why then has the minute eutherian egg evolved such a prominent coat apparently so problematic for the fertilizing spermatozoon? Composed of three or four interlinked glycoproteins, the zona does prevent the adhesion of the embryo to the oviduct epithelium, and it often constitutes the barrier to polyspermy and later maintains an ordered cohesion of the early blastomeres until compaction takes place. However, it is in the uterine phase that its thickness and elasticity seem to come into play. In contrast to the zona's early demise in marsupials (Selwood 2000), these characteristics of the eutherian zona allow it to persist to a species-variable degree as a progressively thinning 

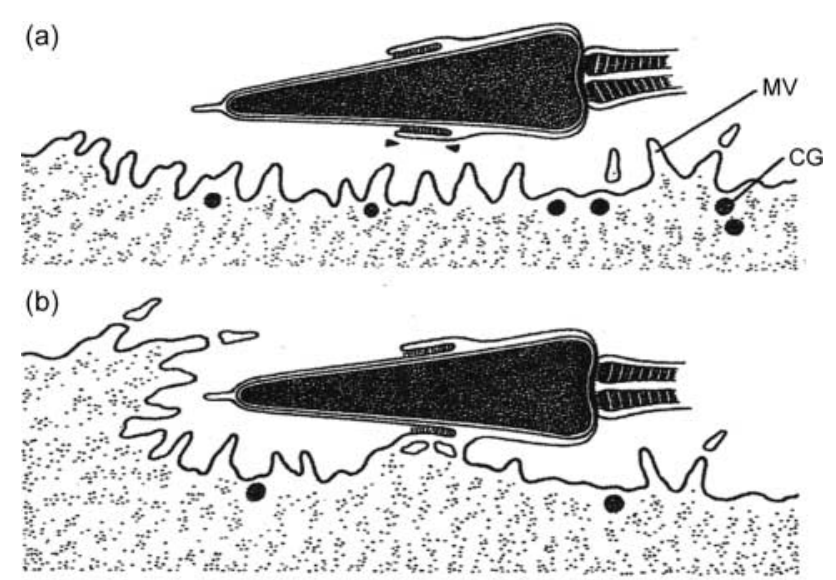

(c)
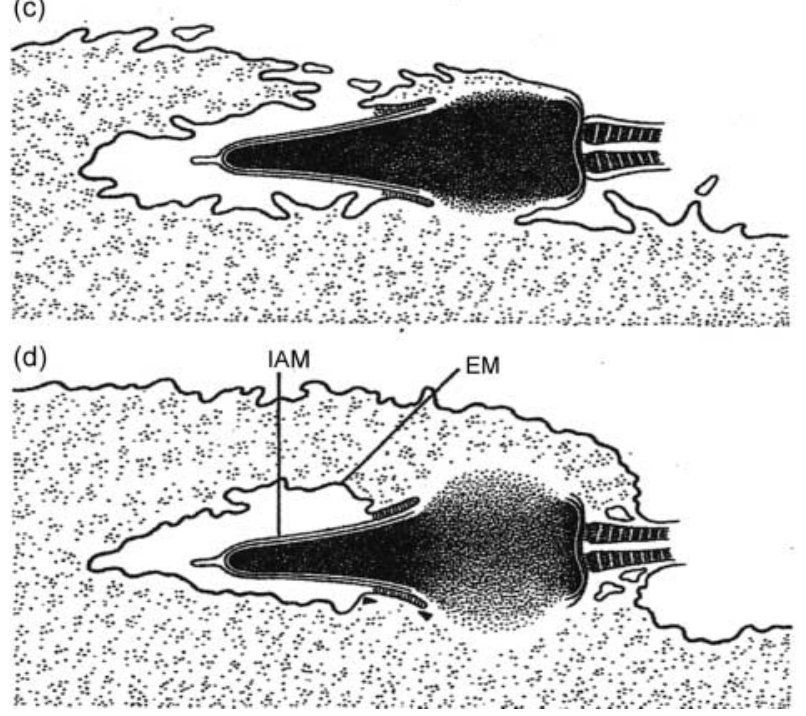

Figure 1 Sequence of events involved in the fusion and incorporation of the fertilizing spermatozoon in a majority of eutherians. Once it reaches the perivitelline space (a), the spermatozoon fuses with the oolemma by way of a limited region of plasmalemma overlying the acrosome's intact equatorial segment (delineated by arrowheads) (b). Fusion stimulates the exocytosis of cortical granules, and the beginning of decondensation in the post-nuclear region begins as the egg cortex engulfs the anterior sperm head, bounded by the persistent inner membrane of the acrosome (c). When sperm incorporation is complete (d), the anterior head region is encased by internalized oolemma. $M V$, egg microvillus; CG, cortical granule; IAM, inner acrosomal membrane; EM, egg plasma membrane.

coat able to stretch and persist as the blastocyst expands. While it is often shed only shortly before implantation, in cattle, sheep, horses and pigs its eventual shedding is followed by further blastocyst growth.

\section{Consequences for the male reproductive tract}

A variety of evidence suggests that the adaptation of spermatozoa for fertilization in the eutherian mode has influenced events in the male concerned with sperm formation and maturation and indirectly with sperm storage and hence sperm capacitation and scrotal evolution as well.

\section{Sperm maturation}

Although limited to studies in only a few subtherian vertebrates, the present picture suggests that the adoption of internal fertilization selected for several sperm features related to issues faced in the female reproductive tract. These features include utilization, to a species-variable degree, of both oxidative phosphorylation and glycolysis; a vermiform sperm head; a variable appearance of skeletal elements in the sperm tail; and generally maturation of the ability to fertilize only after leaving the testis. However, this maturation process appears to involve only the sperm's capacity for progressive motility and a modification of its surface by non-glycosylated proteins (Bedford 1979, Depeiges \& Dufaure 1983, Esponda \& Bedford 1987, Morris et al. 1987).

Compared with those in subtherian vertebrates, both spermiogenesis and the epididymal phase in therian mammals appear to be more elaborate in ways that often can be linked to the particular challenges that their spermatozoa face at fertilization. In the testis, with rare exceptions (Dorman et al. 2014), novel elements in eutherian sperm head morphogenesis that are related to this include formation of the acrosome's insoluble matrix and equatorial segment, transition to a cysteine-rich protamine and perinuclear matrix and, not least, a consistent flattening of the nucleus. The subsequent epididymal phase appears to be more complex than that in subtherian vertebrates with regard to the stabilization of the sperm head, reorganization of the acrosome's content, sperm motility and remodelling of the sperm plasmalemma.

A novelty of epididymal maturation that presents as an adaptation to the challenge of penetrating the zona is the rigidity of the eutherian sperm head. Alone among higher vertebrates (Bedford \& Calvin 1974), much or all of this rigidity is established during epididymal passage by extensive -S-S- cross-linking within the cysteine-rich protamine and perinuclear theca, which serves to rigidify the sperm head. Together with its flat shape, the resulting stiffness would appear to facilitate the sperm head's oscillating or 'scything' thrust used in penetrating the thickness of the zona pellucida.

A second novelty often seen during epididymal maturation is a visible remodelling of the apex of the acrosome. Perhaps reflecting a reorganization of its content (Yoshinaga \& Toshimori 2003, Olsen et al. 2004, Buffone et al. 2008), although not visible in the rat or in man, this has been observed in the guinea pig, chinchilla, loris, rhesus monkey, rabbit, hyrax and white-tailed rat (Mystromys albicaudatus). As noted, the eutherian acrosome is not merely a bag of enzymes in that the fenestrated carapace usually first tethers the sperm head to the zona. The persistence of this 
membrane complex suggests an adaptation that not only maintains the sperm head's contact with a resistant zona, but may also position putative ancillary binding elements such as proacrosin and zonadhesin exposed by the AR. In the case of the porcine acrosome, for example, proacrosin undergoes a redistribution to the apical ridge (Puigmule et al. 2011), a locus that may favour its role as an element in zona binding.

With regard to motility, experiments in a handful of species involving the ligation of the efferent ducts indicate that the capacity for functional motility cannot develop within the eutherian testis. But, unlike the -S-S- crosslinking and the acrosomal modifications discussed above, it is not at all clear why this aspect should have come to depend on the epididymis or exactly what is involved. It is possible that the need to later develop hyperactivated motility on capacitation has been a complication influencing the dependence of this aspect on the epididymis. At all events, in accordance with observations that increased cAMP levels parallel a reduction in certain maturing sperm tail phosphatases (Vijayrhagavan et al. 1996), permeabilization of immature hamster spermatozoa in the presence of cAMP and ATP markedly elevated their motility (Mohri \& Yanagimachi 1980). Thus, certain modifications in the sperm plasmalemma may be integral to motility maturation, though these appear to be distinct from the surface changes involved in zona binding (Bedford 1967).

I first observed epididymal change in the sperm surface in the electrophoretic behaviour of rabbit spermatozoa, and later this principle was confirmed with visual markers such as cationized ferric colloid, lectins and, more recently, immunocytochemistry. However, as noted above, only in therian mammals is there evidence in this regard for a significant involvement of glycoproteins both their loss and neo-expression - as surface or integral components of the sperm plasmalemma in the establishment of zona binding (e.g. Moore (1981)). While sperm surface changes during epididymal passage in therian mammals were first considered only in terms of glycoproteins that probably bear on sperm/zona binding, it is clear that they also involve changes in the plasmalemma's sterol content that can vary according to species (Cross 1998). However, epididymal modifications of the sperm plasmalemma impinge on more than the acquisition of motility and sperm head binding to the zona. For example, underpinned by a fusion factor - Izumo (Inoué et al. 2005) - CRISP1 acquired by the sperm surface in the upper epididymis conditions the final state of the equatorial segment's membrane involved in gamete fusion (Cohen et al. 2011). Not least, epididymal change in the sperm surface has implications for more than fertilization per se. As discussed below, it also appears to be integral to the mechanisms involved in sperm storage in the cauda epididymidis and, directly linked to that, the eventual need for capacitation in the female reproductive tract.

\section{Sperm storage in the cauda epididymidis}

The therian epididymis is a bifunctional organ that supports not only the maturation of spermatozoa, but also their storage in the terminal cauda. In principle, the cauda epididymidis optimizes the ability to deliver several sperm-rich ejaculates within a brief period, with this function being regulated by androgens and very often by the low temperature of the scrotum. There appears to be no similar dedicated storage site in the reptiles, non-passerine birds and a monotreme that we have studied, and in considering why this function has evolved in therian mammals, a number of factors suggest themselves. For example, unlike the mere $24 \mathrm{~h}+$ required in the Japanese quail (Clulow \& Jones 1982), spermatozoa are wafted back and forth within a duct segment of the eutherian epididymis and their transport through it requires more than a week, regardless of great variation in its length according to species. This reduces the potential for sperm replacement after ejaculation, and as seen in comparing the rat and quail (Clulow \& Jones 1982), not only are daily sperm production rates in therian mammals relatively low (Roosen-Runge 1977), but mature spermatozoa also remain functional for no more than \pm 5 days if withheld above the cauda. The possibility that slow transport, modest production and viability issues determined the evolution of the cauda's storage function is supported by the picture in passerine birds that have evolved such a sperm store within a scrotum-like protuberance (Wolfson 1954). Exemplified by the song sparrow Melospiza melodia, the testes produce a relatively low number of spermatozoa, which trickle passively through a ciliated duct to complete a more complex maturation process in the terminal glomus (see Bedford (1979)).

In considering the support for spermatozoa in the cauda, it seems significant that in a variety of mammals the sperm plasmalemma becomes 'coated' in the upper cauda by certain GPI-linked macromolecules first secreted there (Dacheux \& Voglmayr 1983, Thomas et al. 1984, Rifkin \& Olson 1985, Garcia et al. 1988, Derr et al. 2001). However, although macromolecules such as 'HIS protein' and 'CD52' have been designated as 'maturation associated', most spermatozoa develop the ability to fertilize before such macromolecules are acquired. Therefore, it seems likely that such molecules act rather to facilitate the storage of the spermatozoa and, as argued below, they may underlie or at least contribute to the need for capacitation in the female reproductive tract (see Yeung et al. (2000)).

\section{Sperm capacitation}

The term 'capacitation' was coined by C R Austin to describe a functional change in the eutherian spermatozoon undergone in the female reproductive tract that confers on it the ability to fertilize immediately. Although 
it is often stated that epididymal maturation brings about the ability to undergo capacitation, it appears rather that capacitation is necessitated by the state of the sperm plasmalemma established in the cauda, with some of the preliminaries for this initiated more proximally (Rifkin \& Olson 1985). Thus, spermatozoa fertilize sooner if recovered before they enter the cauda region (pigs - Hunter et al. (1986)) or come from a temperaturesuppressed cauda (hamsters - Bedford \& Yanagimachi (1991)). The fact that spermatozoa acquire a further complement of macromolecules in the upper cauda and so finally have developed the ability to fertilize suggests that this acquisition is related to their viability there - in a sense placing them in a physiological 'cocoon'. Since capacitation involves a species-variable loss of sperm membrane cholesterol and specific glycoproteins that activate key ion channels in the plasmalemma, capacitation would appear to constitute a necessary reversion from a stabilized state related to caudal storage to one that enables the $A R$ and development of hyperactivated motility.

\section{The scrotum}

Many pointers suggest that the regulated storage function of the cauda epididymidis has also been the prime mover in the evolution of the scrotal state. A focus on the temperature sensitivity of descended testes has not resolved the question as to the adaptive significance of the scrotum, which is restricted to therian mammals and, as a paracloacal protuberance, many passerine birds. In fact, the eutherian testis operates according to species in anatomical locations that range from the abdomen to the inguinal region to the pendulous scrotum and so over a temperature range of $\sim 6{ }^{\circ} \mathrm{C}$ (Carrick \& Setchell 1977). In a small subset (testiconda) that includes the elephant, hyrax, golden mole, tenrec, elephant shrew and some marine mammals, the testis and epididymis function at deep body temperature within the abdominal cavity. As a secondary adaptation, even fully scrotal testes have on occasion come to tolerate a high ambient temperature of the environment equivalent to that of the body (Bronson \& Heideman 1993). Moreover, the testis' reaction to temperature is not all-or-none: in two cryptorchid models - the musk shrew and the degu elevation of their inguinal testes to the body cavity (raising their temperature by $\pm 2{ }^{\circ} \mathrm{C}$ ) brought about only a partial disruption of spermatogenesis and continued production of some morphologically normal spermatozoa (Bedford et al. 1982). Thus, the evolution of the scrotum does not reflect some fundamental incompatibility between body temperature and spermatogenesis.

It seems germane in asking 'why a scrotum', that this development appears only in conjunction with the cauda epididymidis as a regulated sperm storage site, i.e. in eutherian and marsupial mammals and many passerine birds. In other than the testiconda, the
U-configuration of the epididymis and vas deferens serves to place the cauda in the coolest location, often to protrude well beyond and at a temperature below that of the adjacent testis. This is illustrated to an extreme degree in vespertilionid bats in which an interfemoral membrane provides support for the cauda to extend far beyond the semi-inguinal testis. Equally significant, in a diverse variety of mammals whose scrotal testes are furred, the adjacent region overlying the cauda displays a circumscribed baldness (Bedford 1978). This configuration, as well as occasional examples of discrete pigmentation over the cauda (e.g. bush buck), points to a design for preferential cooling of the cauda epididymidis. The end result of this arrangement in species such as the rat is a cauda temperature of $\sim 4{ }^{\circ} \mathrm{C}$ below that of the adjacent scrotal testis (Brooks 1973), a differential maintained in part in this species by an insulating fat pad between them. On the other hand, it seems significant that in no case does the scrotal arrangement favour the cooling of the testis over the cauda epididymidis.

In considering the scrotum from this point of view, it is interesting that subjection of the scrotal epididymis to body temperature does not inhibit sperm maturation but devastates the sperm storage function. The latter effect is reflected not only in the changed protein and ionic profiles of the cauda's environment, but also in a reduction of its duct length and diameter - and so its carrying capacity. Thus, in rats with epididymides reflected to the abdomen for $3+$ weeks, despite normal testes and sperm numbers in the caput, we observed that repetitive ejaculation to sperm exhaustion produced only $25 \%$ of the number in an ejaculation sequence from normal rats.

While such observations make a case for linking scrotal evolution to the function of the cauda, if temperature sensitivity of the testis has no adaptive significance per se, why does it descend and why does the extent of this differ so widely across species? Given the present focus, I suggest that testis descent provides a means for the cauda epididymidis to 'piggyback' to an inguinal or fully scrotal situation and that the sensitivity of the germinal epithelium to body temperature in scrotal species merely reflects a secondary adaptation of its metabolic machinery to function optimally at the temperature of its location for that species (e.g. Ewing \& Schanbacher (1970)). As for the spectrum of anatomical variations, the size of the testis and character of the scrotum have probably been affected by several factors in the course of mammalian evolution. However, in assessing a wide range of mammals, Freeman (1990) made the important correlation that internal testes tend to be larger per unit body mass and that relative testis size generally declines according to the degree of descent, with the cauda being correspondingly more prominent. Since the rate of sperm production is broadly related to testis size, it may be that descent/exteriorization represents something of a trade-off, with a lower 
level of sperm production offset by a compensatory capacity for sperm storage that maximizes the number immediately available for ejaculation.

\section{Comment}

In contemplating what has determined the characteristics of reproductive systems, the recent literature invokes a process of adaptive co-evolution between the male and female genitalia in groups ranging from Drosophila to waterfowl, with sexual conflict and possibly sperm competition as other elements. In the case of eutherian mammals, however, the present evidence suggests that adaptive novelties in the mode of fertilization are a fallout rather of the character of the egg - in turn giving rise to others that include morphogenesis of the sperm head, features of epididymal sperm maturation and differentiation of a sperm storage function of the cauda epididymidis that bears on both the need for sperm capacitation and scrotal evolution. Such conclusions are supported throughout particularly by a few striking comparative variants on the general pattern.

It has taken many years to appreciate the underlying significance in Eutheria of the special features of the sperm head and of fertilization. The cumulus oophorus presents as a dense cell mass essential for the induction of the AR in putatively primitive shrews and probably a few other genera, but this has become characterized more often during the mammalian radiations by a matrix that is not essential for fertilization per se but abets in snagging the few spermatozoa at the fertilization site and/or possibly at ovulation in egg transport to the tubal ampulla. However, whether the AR begins within the cumulus or on the zona, the interaction thereafter involves several adaptive sperm features established during spermiogenesis and epididymal passage that can be linked to the challenge of penetrating the zona pellucida.

A red flag for understanding the mechanisms involved in zona penetration is the unique configuration of gamete fusion (Fig. 1). The 'equatorial' fusion site appears to have evolved in the face of an unusual physicality of the IAM, which is a trait probably related to shear forces in penetrating the zona. That this penetration involves a major physical component can be inferred from other unusual features of sperm head design - a flat profile and an -S-S- bond-based stiffness that permit scything-like oscillations of the head within a narrowly defined slit, powered by the hyperactivated tail beat. In considering the evidence for zona lysis, such a role for the historical favourite, acrosin, seems unlikely now for the several reasons discussed, but whether or not penetration in Eutheria is typically a non-lytic event remains to be resolved. Although the acrosome's content is discarded before penetrating a zona relatively resistant to proteases, the concept of zona lysis has been hard to abandon, as shown by recent claims for various other candidate enzymes, referenced above. However, comparative observations perhaps provide further evidence for the non-lytic version. For example, the rabbit acrosome has a smaller complement of several enzymes (acrosin, hyaluronidase, hexosaminidase and arylsulfatase A) than the marsupial opossum acrosome (Rodger \& Young 1981), which possesses a thin protease-sensitive zona. This points to an absence of any compensatory selection for acrosomal enzymes with evolutionary hypertrophy of the eutherian zona. Moreover, and illogically for zona lysis, the environment of the fallopian tube renders the zona more protease-resistant in a variety of species excepting man (Mondejar et al. 2013). Finally, in a few southeast Asian rodents exemplified by $B$. indica, a thinner putatively protease-sensitive zona pellucida is matched by a sperm head that is round in profile and has minimal -S-S- cross-linking and a large rostral acrosome lacking an equatorial segment (Dorman et al. 2014) - all departures from the eutherian norm consistent with a pattern of penetration by zona lysis.

Such adaptations in eutherian spermatozoa are also reflected as new elements in the process of sperm preparation in the male. This is seen during spermiogenesis in the final transition to a uniquely cysteine-rich protamine, in the flat profile of the sperm head, and in the appearance of an insoluble component of the acrosomal matrix and of the equatorial segment of the acrosome. Further aspects in the epididymis include a subtle reorganization of the acrosomal matrix that may ensure at least an apical marshalling of intra-acrosomal contributors to zona binding (Puigmule et al. 2011) and the possibility for the sperm nucleus to emerge from the reacted acrosome carapace during the first step of zona penetration (Olsen et al. 2004). Other novel epididymal events that are related to this interaction include sperm head rigidification based on -S-S- cross-linking within the nucleus and perinuclear matrix and proteoglycan modifications of the sperm plasmalemma involved in the initiation of sperm/zona binding. In accordance with the last point, our efforts to identify glycan-related modifications of the sperm surface were not successful with lectins at least in Suncus murinus - a shrew whose acrosome is shed long before reaching the zona and in which zona binding is effected by barbs on the perforatorium. In summary, observations in a variety of vertebrates suggest that the pattern of sperm maturation in the epididymis may have evolved in two phases - a first simpler subtherian mode related to issues that spermatozoa face in the female reproductive tract, complicated then by adaptations to the challenges met in fertilizing the eutherian egg.

Returning to the state of the sperm plasmalemma, its coating by GPI-linked macromolecules on entering the proximal cauda and the appearance of receptors for them in the upper epididymis (Rifkin \& Olson 1985) are probably related rather to the survival of spermatozoa in 
the cauda epididymidis. In eutherian mammals at least, storage function appears to enable the production of repetitive sperm-rich ejaculates despite a limited potential for rapid sperm replacement probably brought about not only by a relatively modest production but also by their slow epididymal transport perhaps related to the complexity of sperm maturation there. Together with the brief life span of mature spermatozoa withheld above the cauda, such issues may have driven the evolution of the specialised caudal storage site, the state that prolongs sperm viability there, bringing a subsequent need for their capacitation in the female reproductive tract.

Finally, returning to the scrotum. As well as the phenomenon of capacitation, a second consequence of the evolution of the cauda sperm storage function appears to be the evolution of the scrotal state. Our experiments in the rat, as an example, demonstrate that temperature suppression of the cauda epididymidis alone not only removes its ability to prolong sperm viability, but also results in $75 \%$ fewer spermatozoa in an ejaculation sequence. Thus, although sperm maturation is not suppressed by it, the storage function is as vulnerable to body temperature as is spermatogenesis. Why a cooler state should act to enhance the cauda's potential for sperm storage is not clear, but, whatever the explanation, three points are critical to understanding this arrangement. First, a scrotum has developed only in conjunction with a temperature-regulated sperm storage function: to wit, in therian mammals and passerine birds. Second, in the eutherians examined so far, both the scrotal pelage and location of the cauda often favour its cooling over that of the adjacent testis, but never the reverse. Third, although spermatogenesis in the scrotal testis is typically disrupted, it is clear that temperature is not a sacrosanct issue for the therian testis as such in that this can function over a range, each temperature set according to species (Carrick \& Setchell 1977). Given the impression that external migration of the testis helps the cauda of the associated epididymis to 'ride' with and often beyond it to a cool location, the notorious sensitivity of the scrotal testis may merely reflect a secondary adaptation to function optimally at the temperature of its location.

On the other hand, the idea that the sperm storage function of the cauda has driven the development of the scrotal state is perhaps vitiated by species variation in testis size, the extent of its external migration, the prominence of the cauda and, not least, the scrotal pelage. It is likely that this diversity has been affected by several factors. In man, for instance, the modesty of the cauda may be exaggerated further by the elevated temperature brought about by inguinal clothing. In marine mammals, issues of temperature regulation and streamlining may come into play, and in many species, development of the cauda and so sperm delivery have probably been affected not only by the common practice of polygyny (i.e. the potential need for repetitive ejaculation), but also by different patterns of sperm utilization within the female reproductive tract. However, an explanation for the range in both the size and position of the eutherian testis versus that of the cauda is suggested by the regression analysis of Freeman (1990) in a wide range of species. While it is incorrect to conclude, as he does, that internal testes produce 'lowquality' spermatozoa, Freeman's study provides evidence for a trade-off between the relative size of the testis and so sperm production, on the one hand, and the potential for sperm storage, on the other. His figures indicate that internal testes tend to be relatively larger and produce more spermatozoa, whereas scrotal testes are relatively smaller but with an optimal cauda sperm store compensating for this deficit. In other words, lowtemperature-enhanced epididymal storage has reduced the pressure to produce spermatozoa without compromising the number ejaculated. This paradigm is epitomized by the situation in the rat compared with that in the similarly sized Japanese quail (Clulow \& Jones 1982), both of which can ejaculate some 300 million spermatozoa within a limited period. The quail achieves this by producing a greater number from a larger testis with rapid epididymal transport, whereas the smaller scrotal testis of the rat produces fewer spermatozoa with slower throughput, but equal numbers in an ejaculate series, thanks to the cauda's sperm store.

\section{Declaration of interest}

The author declares that there is no conflict of interest that could be perceived as prejudicing the impartiality of the review reported.

\section{Funding}

This review did not receive any specific grant from any funding agency in the public, commercial or not-for-profit sector.

\section{References}

Anderson E, Hoppe EC, Whitten WK \& Lee GS 1975 In vitro fertilization and early embryogenesis: a cytological analysis. Journal of Ultrastructure Research 50 231-252. (doi:10.1016/S00225320(75)80054-2)

Avelia MA, Xiong B \& Dean J 2013 The molecular basis of gamete recognition in mice and humans. Molecular Human Reproduction 19 279-289. (doi:10.1093/molehr/gat004)

Baba T, Azuma S, Kashiwabara S \& Toyoda Y 1994 Sperm from mice carrying a targeted mutation of the acrosin gene can penetrate the oocyte zona pellucida and effect fertilization. Journal of Biological Chemistry 269 31845-31849.

Barros C, Bedford JM, Franklin LE \& Austin CR 1967 Membrane vesiculation as a feature of the mammalian acrosome reaction. Journal of Cell Biology 34 C1-C5. (doi:10.1083/jcb.34.3.C1)

Bedford JM 1967 Effects of duct ligation on the fertilizing ability of spermatozoa from different regions of the rabbit epididymis. Journal of Experimental Zoology 166 271-282. (doi:10.1002/jez.1401660210) 
Bedford JM 1972 An electron microscope study of sperm penetration into the rabbit egg after natural mating. American Journal of Anatomy 133 213-254. (doi:10.1002/aja.1001330206)

Bedford JM 1978 Anatomical evidence for the epididymis as the prime mover in the evolution of the scrotum. American Journal of Anatomy 133 213-254. (doi:10.1002/aja.1001330206)

Bedford JM 1979 Evolution of the sperm maturation and sperm storage functions of the epididymis. In The Spermatozoon: Maturation, Motility, Surface Properties and Comparative Aspects, pp 7-21. Eds DW Fawcett\& JM Bedford. Baltimore-Munich: Urban and Schwartzenberg.

Bedford JM \& Calvin H 1974 The occurrence and possible functional significance of -S-S- crosslinks in sperm heads, with particular reference to eutherian mammals. Journal of Experimental Zoology 188 137-156. (doi:10.1002/jez.1401880203)

Bedford JM \& Cross NL 1978 Normal penetration of rabbit spermatozoa through a trypsin and acrosin-resistant zona pellucida. Journal of Reproduction and Fertility 54 385-392. (doi:10.1530/jrf.0.0540385)

Bedford JM \& Yanagimachi R 1991 Epididymal storage at abdominal temperature reduces the time for capacitation of hamster spermatozoa. Journal of Reproduction and Fertility 91 403-410. (doi:10.1530/jrf.0. 0910403)

Bedford JM, Moore HD \& Franklin LE 1979 Significance of the equatorial segment of the acrosome of the spermatozoon in eutherian mammals. Experimental Cell Research 119 119-126. (doi:10.1016/ 0014-4827(79)90341-0)

Bedford JM, Berrios M \& Dryden GL 1982 Biology of the scrotum IV. Testis location and temperature sensitivity. Journal of Experimental Zoology 224 379-388. (doi:10.1002/jez.1402240311)

Bedford JM, Mock OB \& Goodman SM 2004 Novelties of conception in insectivorous mammals (Lipotyphla), particularly shrews. Biological Reviews of the Cambridge Philosophical Society 79 891-909. (doi:10.1017/S1464793104006529)

Bronson FH \& Heideman PD 1993 Failure of cryptorchidism to suppress fertility in a tropical rodent. Biology of Reproduction 48 1354-1359. (doi:10.1095/biolreprod48.6.1354)

Brooks DE 1973 Epididymal and testicular temperature in the unrestrained conscious rat. Journal of Reproduction and Fertility 35 157-160. (doi:10.1530/jrf.0.0350157)

Buffone MG, Foster JA \& Gerton GL 2008 The role of the acrosomal matrix in fertilization. International Journal of Developmental Biology 52 511-522. (doi:10.1387/ijdb.072532mb)

Carrick FN \& Setchell BP 1977 The evolution of the scrotum. In Reproduction and Evolution, pp 165-170. Eds JH Calaby \& CH Tyndale-Biscoe. Canberra: Australian Academy of Science.

Clulow J \& Jones RC 1982 Production, transport, maturation, storage and survival of spermatozoa in the male Japanese quail, Coturnix coturnix. Journal of Reproduction and Fertility 64 259-266. (doi:10.1530/jrf.0. 0640259)

Cohen DJ, Ellerman DA, Busso D, Morgenfeld MM, Kasahara M \& Cuasnicu PS 2011 Evidence that human epididymal protein ARP plays a role in gamete fusion through complementary sites on the surface of the human egg. Biology of Reproduction 65 1000-1005. (doi:10.1095/ biolreprod65.4.1000)

Cross NL 1998 Role of cholesterol in sperm capacitation. Biology of Reproduction 59 7-11. (doi:10.1095/biolreprod59.1.7)

Dacheux J-L \& Voglmayr JK 1983 Sequences of sperm cell differentiation and its relationship to exogenous fluid proteins in the ram epididymis. Biology of Reproduction 29 1033-1047. (doi:10.1095/ biolreprod29.4.1033)

Depeiges A \& Dufaure JP 1983 Binding to spermatozoa of a major soluble protein secreted by the epididymis of the lizard Lacerta vivipara. Gamete Research 7 401-406. (doi:10.1002/mrd.1120070411)

Derr P, Yeung CH, Cooper TG \& Kirchhoff C 2001 Synthesis and glycosylation of CD 52, the major 'maturation associated' antigen on rat spermatozoa in the cauda epididymidis. Reproduction 121 435-446. (doi:10.1530/rep.0.1210435)

Dorman F, Balsamo P, Leigh C \& Breed WG 2014 Co-evolution of gametes of the greater bandicoot rat, Bandicota indica - a murine rodent from South-East Asia. Acta Zoologica. In press. (doi:10.1111/azo.12036)

Dziuk PJ \& Dickman Z 1965 Sperm penetration of the zona pellucida of the pig egg. Journal of Experimental Biology 41 177-182.
Esponda P \& Bedford JM 1987 Post-testicular changes in the reptile sperm surface with particular reference to the snake, Natrix fasciata. Journal of Experimental Zoology 241 123-132. (doi:10.1002/jez.1402410115)

Ewing LL \& Schanbacher LM 1970 Early effects of experimental cryptorchidism on the activity of selected enzymes in rat testes. Endocrinology 87 129-134. (doi:10.1210/endo-87-1-129)

Ferrer M, Rodriguez H, Zara L, Yu Y, Xu W \& Oko RM 2012 MP2 and acrosin are major proteinases associated with the inner acrosomal membrane and may cooperate in sperm penetration of the zona pellucida during fertilization. Cell and Tissue Research 349 881-895. (doi:10.1007/s00441-012-1429-1)

Freeman S 1990 The evolution of the scrotum: a new hypothesis. Journal of Theoretical Biology 145 429-445. (doi:10.1016/S00225193(05)80479-5)

Garcia C, Regalado F, Lopez de Haro MS \& Nieto A 1988 Ultrastructural localization of epididymal secretory proteins associated with the surface of spermatozoa from rabbit cauda epididymis. Histochemical Journal 20 708-714. (doi:10.1007/BF01002751)

Green DPL 1997 Three-dimensional structure of the zona pellucida. Reviews of Reproduction 2 147-156. (doi:10.1530/ror.0.0020147)

Huang TTF \& Yanagimachi R 1985 Inner acrosomal membrane of mammalian spermatozoa; its properties and possible functions in fertilization. American Journal of Anatomy 174 249-268. (doi:10.1002/ aja.1001740307)

Huang TTF, Fleming AD \& Yanagimachi R 1981 Only acrosome-reacted spermatozoa can bind to and penetrate zona pellucida: a study using the guinea pig. Journal of Experimental Zoology 217 287-290. (doi:10.1002/ jez.1402170215)

Hunter RHF, Holtz W \& Henfrey PJ 1986 Stabilising role of epididymal plasma in relation to capacitation time of boar spermatozoa. Animal Reproduction Science 1 161-166. (doi:10.1016/0378-4320(78)90024-6)

Inoué N, Ikawa M, Isotani A \& Okabe M 2005 The immunoglobulin superfamily protein Izumo is required for sperm to fuse with eggs. Nature 434 234-238. (doi:10.1038/nature03362)

Inoué N, Satouh Y, Ikawa M, Okabe M \& Yanagimachi R 2011 Acrosomereacted mouse spermatozoa recovered from the perivitelline space can fertilize other eggs. PNAS 108 20008-200011. (doi:10.1073/pnas. 1116965108)

Jin M, Fujiwara E, Kakiuchi Y, Okabe M, Satouh Y, Baba SA, Chiba K \& Hirohashi N 2011 Most fertilizing spermatozoa begin their acrosome reaction before contact with the zona pellucida during in vitro fertilization. PNAS 108 4892-4896. (doi:10.1073/pnas. 1018202108)

Jansen S, Quigley M, Reik W \& Jones R 1995 Analysis of polysulfate binding domains in porcine proacrosin, a putative zona adhesion protein from mammalian spermatozoa. International Journal of Developmental Biology 39 501-510.

Kim E, Yamashita M, Kimura M, Honda A, Kashiwabara S \& Baba T 2008 Sperm penetration through cumulus mass and zona pellucida. International Journal of Developmental Biology 52 677-682. (doi:10.1387/ijdb.072528ek)

Klemm U, Muller-Esterl W \& Engel W 1991 Acrosin, the peculiar spermspecific serine protease. Human Genetics 87 635-641. (doi:10.1007/ BF00201716)

Kopecny V \& Flechon JE 1987 Ultrastructural localisation of labelled acrosomal glycoproteins during in vivo fertilization in the rabbit. Gamete Research 17 35-42. (doi:10.1002/mrd.1120170105)

Kuzan FB, Fleming AD \& Seidel GE 1984 Successful fertilization in vitro of fresh intact oocytes by perivitelline (acrosome-reacted) spermatozoa of the rabbit. Fertility and Sterility 41 766-770.

Lewis CA, Talbot CF \& Vacquier VD 1982 A protein from abalone sperm dissolves the egg vitelline layer by a non-enzymatic mechanism. Developmental Biology 92 227-239. (doi:10.1016/00121606(82)90167-1)

Llanos M, Vigil P, Slagado AM \& Morales P 1993 Inhibition of the acrosome reaction by trypsin inhibitors and prevention of penetration of spermatozoa through the human zona pellucida. Journal of Reproduction and Fertility 97 173-178. (doi:10.1530/jrf.0.0970173)

Meizel S 1984 The importance of hydrolytic enzymes to an exocytotic event. Biological Reviews of the Cambridge Philosophical Society 59 125-157. (doi:10.1111/j.1469-185X.1984.tb00404.x) 
Mohri H \& Yanagimachi R 1980 Characteristics of motor apparatus in testicular, epididymal and ejaculated spermatozoa: a study using demembranated sperm model. Experimental Cell Research 127 191-196. (doi:10.1016/0014-4827(80)90426-7)

Mondejar I, Aviles M \& Coy P 2013 The human is an exception to the evolutionarily-conserved phenomenon of pre-fertilization zona pellucida resistance to proteolysis induced by oviductal fluid. Human Reproduction 28 718-728. (doi:10.1093/humrep/des423)

Moore HDM 1981 Glycoprotein secretions of the epididymis in the rabbit and hamster: localization on epididymal spermatozoa and the effect of specific antibodies on fertilization in vivo. Journal of Experimental Zoology 215 77-85. (doi:10.1002/jez.1402150109)

Moreno RD, Laserre AA \& Barros C 2011 Protease activity involvement in the passage of mammalian sperm through the zona pellucida. Biological Research 44 145-150. (doi:10.4067/S0716-97602011000200006)

Morris SA, Howarth B, Crim JW, Rodriguez de Cordoba S, Esponda P \& Bedford JM 1987 Specificity of sperm binding Wolffian duct proteins in the rooster and their persistence on spermatozoa in the female host glands. Journal of Experimental Zoology 242 189-198. (doi:10.1002/ jez.1402420210)

Olsen GE, Winfrey VP, Bi M, Hardy DM \& Nagdas SK 2004 Zonadhesin assembly into the hamster sperm acrosome matrix occurs by distinct targeting strategies during spermiogenesis and maturation in the epididymis. Biology of Reproduction 71 1128-1134. (doi:10.1095/ biolreprod.104.029975)

Overstreet JW \& Bedford JM 1974 Comparison of the penetrability of the egg vestments in follicular oocytes, unfertilized and fertilized ova of the rabbit. Developmental Biology 41 185-192. (doi:10.1016/00121606(74)90292-9)

Puigmule M, Fabrega A, Yeste M, Bonet S \& Pinart E 2011 Study of the proacrosin/acrosin system in epididymal, ejaculated and in vitro capacitated boar spermatozoa. Reproduction, Fertility, and Development 23 837-845. (doi:10.1071/RD10345)

Rifkin JM \& Olson GE 1985 Characterization of maturation-dependent extrinsic proteins of the rat sperm surface. Journal of Cell Biology $\mathbf{1 0 0}$ 1582-1591. (doi:10.1083/jcb.100.5.1582)

Rodger JC \& Young RJ 1981 Glycosidase and cumulus dispersal activities of acrosomal extracts from opossum(marsupial) and rabbit (eutherian) spermatozoa. Gamete Research 4 507-514. (doi:10.1002/mrd. 1120040604)

Roosen-Runge EC 1977 The process of spermatogenesis in animals. London: Cambridge University Press.

Selwood L 2000 Marsupial egg and embryo coats. Cells, Tissues, Organs 166 208-219. (doi:10.1159/000016733)

Shur BD 2008 Reassessing the role of protein-carbohydrate complementarity during sperm-egg interactions. International Journal of Developmental Biology 52 703-716. (doi:10.1387/ijdb.082571bs)

Srivastava PN, Adams CE \& Hartree EF 1965 Enzymic action of acrosomal preparations on the rabbit ovum in vitro. Journal of Reproduction and Fertility 10 61-67. (doi:10.1530/jrf.0.0100061)

Sutovsky P 2011 Sperm proteasome and fertilization. Reproduction 142 1-14. (doi:10.1530/REP-11-0041)

Szollosi D \& Hunter RHF 1978 The nature and occurrence of the acrosome reaction in spermatozoa of the domestic pig. Journal of Anatomy 127 $33-41$.
Tardif S, Wilson MD, Wagner R, Hunt P, Gertenstein M, Nagy A, Lobe C, Koop BF \& Hardy DM 2010 Zonadhesin is essential for speciesspecificity of sperm adhesion to the egg zona pellucida. Journal of Biological Chemistry 285 24863-24870. (doi:10.1074/jbc.M110. 123125)

Thibault C \& Dauzier L 1960 "Fertilisines" et fécondation in vitro de l'oeuf de la lapine. Comptes Rendus de l'Acad?mie des Sciences 250 1358-1359.

Thomas TS, Reynolds AB \& Oliphant G 1984 Evaluation of the site of synthesis of rabbit sperm acrosome stabilizing factor using immunocytochemical and metabolic labelling techniques. Biology of Reproduction 30 693-705. (doi:10.1095/biolreprod30.3.693)

Thompson RS, Smith DM \& Zamboni L 1974 Fertilization of mouse ova in vitro - an electron microscope study. Fertility and Sterility 25 222-249.

Vijayrhagavan S, Stephens DT, Trautman K, Smith GD, Khatra B, da Cruz e Silva EF \& Greengard P 1996 Sperm motility development in the epididymis is associated with decreased glycogen synthase kinase-3 and protein phosphatase I activity. Biology of Reproduction 54 709-718. (doi:10.1095/biolreprod54.3.709)

Wolfson A 1954 Sperm storage at lower than body temperature outside the body cavity in some passerine birds. Science 120 68-71. (doi:10.1126/ science.120.3106.68)

Xu H, Liu F, Skraew N, Koppisetty C, Nyholm PG, Carmona E \& Tanphaichitr N 2012 Sperm arylsulfatase A binds to mZP2 and mZP3 glycoproteins in a non-enzymatic manner. Reproduction 144 209-219. (doi:10.1530/REP-11-0338)

Yanagimachi R \& Noda YD 1970a Electron microscope studies of sperm incorporation into the golden hamster egg. American Journal of Anatomy 128 429-462. (doi:10.1002/aja.1001280404)

Yanagimachi R \& Noda YD $1970 b$ Ultrastrucural changes in the hamster sperm head during fertilization. Journal of Ultrastructure Research 31 465-485. (doi:10.1016/S0022-5320(70)90163-2)

Yanagimachi R \& Phillips DM 1984 The status of acrosomal caps of hamster sperm immediately before fertilization in vivo. Gamete Research 9 1-20. (doi:10.1002/mrd.1120090102)

Yeung CH, Schroter S, Kirchhoff C \& Cooper TG 2000 Maturation changes of monkey sperm CD52 and their apparent reversal in capacitation conditions. Molecular Reproduction and Development 57 280-289. (doi:10.1002/1098-2795(200011)57:3<280::AID-MRD10> 3.0.CO;2-5)

Yoshinaga K \& Toshimori K 2003 Organization and modifications of sperm acrosomal molecules during spermatogenesis and epididymal maturation. Microscopy Research and Technique 61 39-45. (doi:10. 1002/jemt.10315)

Zamboni L 1971 Acrosome loss in fertilizing mammalian spermatozoa: a clarification. Journal of Ultrastructure Research 34 401-405. (doi:10. 1016/S0022-5320(71)80081-3)

Received 9 September 2013

First decision 14 October 2013

Revised manuscript received 23 October 2013

Accepted 5 November 2013 